\title{
Effects of Rocket Outgassing on RF Experiments
}

\author{
W. Pfister and J. C. Ulwick \\ Air Force Cambridge Research Laboratories, \\ Office of Aerospace Research, Bedford, Mass., 01731
}

(Received Feb. 9, 1965)

\begin{abstract}
Peculiarities in the results of electron-density measurements with rockets were noticed on several occasions. They were found to be effects of rocket outgassing. When the fuel valves of Aerobee rockets are not shut after burnout, residual fuel leaks out and diffuses into the atmosphere. Electrons attach to the fuel and decrease in concentration while the positive ions are not affected. Outgassing effects were observed on probe experiments flown in rockets as well as on propagation experiments between rocket and ground. Quantitative estimates indicate that the leaking fuel is a very effective attaching agent. Some solid-fuel rockets also show similar effects.
\end{abstract}

\section{Introduction}

Outgassing of rocket fuel after burnout can change the electron density distribution around the rocket and influence the measurements of radio frequency experiments. In fact such effects were observed as early as when the V-2 series were in use. However, at that time they were not recognized properly. Lien et al. [1953] reported on bifurcation of the $E$ layer measured with a pulse delay experiment on several rockets. Subsequently, the apparent bifurcation was explained as an effect of an irregular local deviation from the horizontal stratification [Pfister and Ulwick, 1958]. The manmade nature of the local irregularity was not recognized until rockets were instrumented with probes for the measurement of environmental electron and ion densities. The surprising result was a local deficiency of electrons while the positive ions remained unaffected. The explanation was an outgassing of rocket fuel after burnout [Pfister and Ulwick, 1961].

The following sections describe the observations with the probe experiments on Aerobee rockets in detail. Next follows a somewhat simplified theory on the outgassing effect invoked. The results of the pulse delay experiments are reviewed in terms of the concept of outgassing. Finally, observations on solid-fuel rockets are reported, indicating similar effects.

\section{Probe Observations on Aerobee Rockets \\ 2.1. Instrumentation}

An experiment combining a standing wave impedance probe and a retarding potential probe was flown on two Aerobee 150 rockets with encouraging results, some of which have been published [Pfister, Ulwick, and Vancour, 1961].

The impedance probe is designed for the measurement of electron density and collision frequency. A detailed description of the basic instrument is avail- able in the literature [Haycock and Baker, 1961; Ulwick et al., 1962; Ulwick et al., 1964].

An Aerobee-Hi rocket, numbered $\mathrm{SN}-37$, was launched in 1959 on August 28 at 1140 MST at White Sands, N. Mex. The impedance probe consisted of two completely independent instruments that measured simultaneously at 3 and $7.2 \mathrm{Mc} / \mathrm{s}$, with the impedance going through resonance in both cases. In order to minimize the effect of the ion sheath around the antenna, a symmetrical dipole arrangement was used consisting of a pair of $8-\mathrm{ft}$ whips for each frequency. Loading coils outside the rocket body insured a minimum $R F$ voltage at the feed points and made the free space reactance slightly inductive. For mechanical reasons the loading coils and the antenna wire were completely imbedded in the fiberglass whips. The RF voltage was kept less than 1 V rms.

A second rocket, numbered AA 3.183C, was fired in 1960 on May 24 at 1140 CST from Eglin Air Force Base, Florida. In this rocket the two frequencies used for the impedance probe were 7.2 and $12 \mathrm{Mc} / \mathrm{s}$.

The retarding potential probe flown on both rockets consisted essentially of an arrangement of parallel grids and a target plate. The aperture grid was connected with the skin of the rocket and exposed to the environment after ejection of the nose tip. The potential applied to the auxiliary grid served for the selection of different modes of operation. A sweep potential was applied to the retarding grid and the current was measured at the target plate. A complete description of the experimental technique has been published [Hinteregger, 1960, Ulwick et al., 1962]. For the outgassing studies only the unretarded positive ion mode is of interest.

\subsection{Measurements}

Some of the results of the data analysis for rocket SN-37 have been presented previously [Pfister et al., 


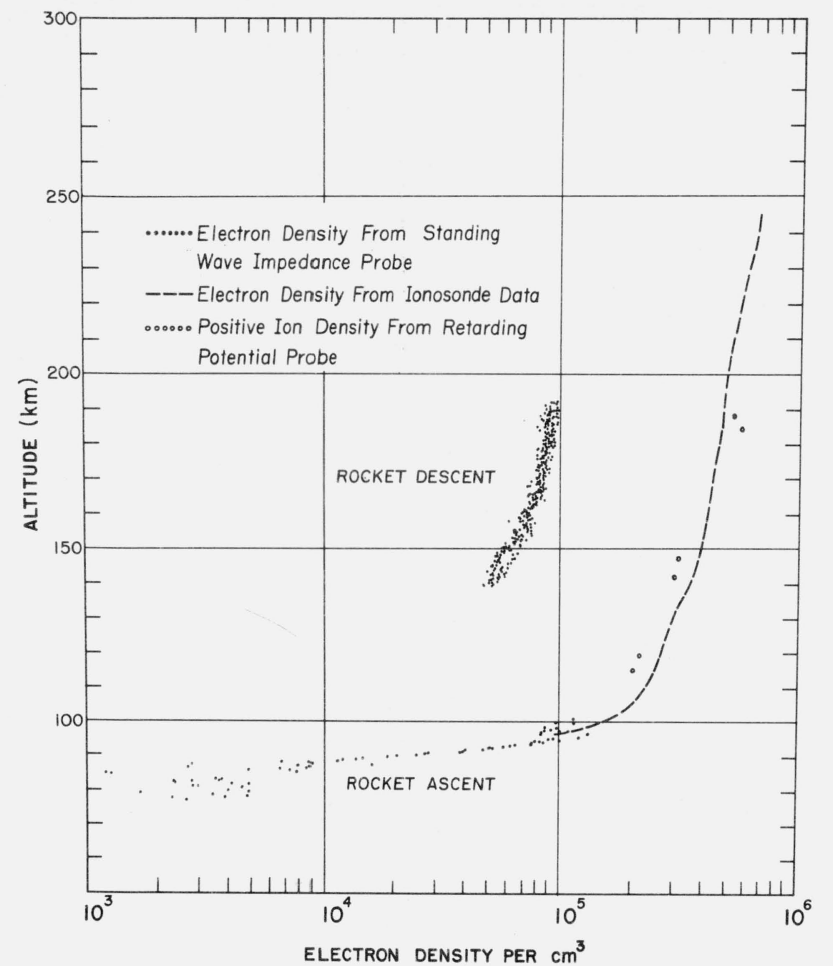

Figure 1. Results from Aerobee $S N-37$, fired 28 August 1959, 1140 MST at White Sands, N. Mex.

1961]. In the meantime, a more detailed data analysis was performed and the results important for the outgassing effect are shown in figure 1 . The electron density was computed from the antenna impedance at $7.2 \mathrm{Mc} / \mathrm{s}$ and plotted as a function of height. The impedance probe was not designed for a wide enough range of electron densities. At a density value of $10^{5} / \mathrm{cm}^{3}$ a complete mismatch of the transmission line occurred and no higher values could be measured. Therefore, it is not surprising that data were lost at a rocket height of $95 \mathrm{~km}$. Completely unexpected was a reappearance of electron density measurements on descent between 180 and $140 \mathrm{~km}$. Data are missing below $140 \mathrm{~km}$ due to telemetry failure. The positive ion density is measured at several height levels on ascent and it agrees essentially with the electron density curve derived from ionosonde data. Based on this evidence, we conclude that the positive ion density equals the unperturbed electron density.

Due to an instrument failure, positive ion densities could not be measured in Aerobee AA3.183C. However, the impedance probe at a frequency of $12 \mathrm{Mc} / \mathrm{s}$ was able to measure the electron density over the entire flight in the ionosphere as shown in figure 2. Judged against the ionosonde data, the impedance probe measures correctly on rocket ascent up to around 130 $\mathrm{km}$. Higher up the measured electron density gradually decreases and reaches $1 / 5$ of the undisturbed density at peak altitude. The descent curve is considerably scattered because the probe at $12 \mathrm{Mc} / \mathrm{s}$

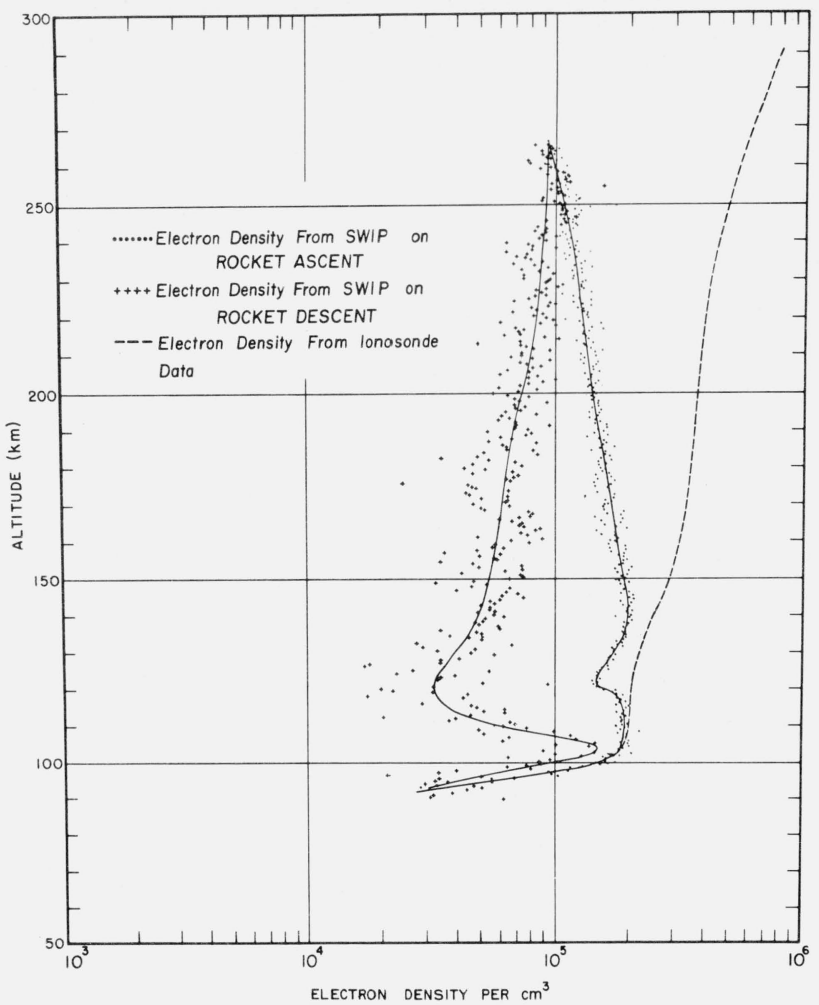

Figure 2. Results from Aerobee AA 3. 183C, fired 24 May 1960, 1140 CST at Eglin Air Force Base, Florida.

reaches a sensitivity limit at $3 \times 10^{4}$ electrons $/ \mathrm{cm}^{3}$ and the measurements at $7.2 \mathrm{Mc} / \mathrm{s}$ could not be used due to a distortion of the standing wave pattern of unknown origin. Nevertheless, descent data in general are a factor 5 below normal until rocket tipover at $105 \mathrm{~km}$ where the probe measures again the undisturbed value.

The interpretation of the data is based on attachment of free electrons to gas which escapes from the rocket. The rocket fuel valves were still left open after burnout. Burnout occurs when the oxidizer is used up while residual fuel is left in the tank. The propellants for an Aerobee rocket consist of a mixture of analine $\left(\mathrm{C}_{6} \mathrm{H}_{5} \mathrm{NH}_{2}\right)$ and furfural alcohol $\left(\mathrm{C}_{4} \mathrm{H}_{3} \mathrm{OCHO}\right)$ as the fuel and red fuming nitric acid as the oxidizer. The residual fuel escapes throughout the flight. When the diffusion velocity of the fuel reaches a value greater than the rocket velocity, the ambient electron density around the antenna is reduced by an attachment process. On descent the antenna is to the rear of the nozzle and thus surrounded by more fuel gas until tipover occurs.

\subsection{Theoretical Considerations}

In order to substantiate this interpretation further, an attempt will be made to compute the decrease in electron density numerically with the use of reasonable assumptions. 
The starting point is the solution of the diffusion equation for a momentary point source

$$
n=\frac{N}{(4 \pi D t)^{3 / 2}} \exp \left\{-\frac{r^{2}}{4 D t}\right\}
$$

where $n$ is the number of particles of the diffusing gas as a function of distance $r$ and time $t ; N$ is the total number of particles released at $t=0$ and $r=0$; and $D$ is the ordinary diffusion coefficient of the diffusing gas in air.

In the present case, the particles are released at a rate $N^{\prime}$ per second from a rocket moving with a velocity $v$ in the $z$ direction. The fuel having a higher pressure than ambient expands quickly before normal diffusion takes place. A simplified model was considered which assumes instantaneous expansion into a Gaussian distribution such that the number density of the fuel at the release point $\left(f n_{0}\right)$ is proportional to the ambient air density $n_{0}$. The particle density $n$ of the fuel is a function of the distance $z$ ahead of the release point and of the lateral distance $\rho$ given according to the model by:

$$
n(z)=\int_{0}^{\infty} \frac{N^{\prime}}{\pi^{3 / 2}\left(r_{0}^{2}+4 D t\right)^{3 / 2}} \operatorname{esp}\left\{-\frac{\rho^{2}+(z+v t)^{2}}{r_{0}^{2}+4 D t}\right\} \mathrm{dt} .
$$

$r_{0}$ is the scale width of the Gaussian distribution and according to the assumption of the model

$$
r_{0}=\sqrt{\frac{N^{\prime}}{\pi v f n_{0}}} .
$$

Numerical computations showed that a model based on diffusion only gives a more satisfactory agreement with the observations in the height range above 120 $\mathrm{km}$ than the more complicated model with the initial expansion or any other simple model based largely on an inviscid flow pattern. Therefore, only the solution of the simplified (2) for $r_{0}=0$ will be discussed:

$$
n(z)=\frac{N^{\prime}}{4 \pi D \sqrt{\rho^{2}+z^{2}}} \exp \left\{-\frac{v}{2 D}\left(z+\sqrt{\rho^{2}+z^{2}}\right)\right\} .
$$

The solution may be written in the form

$$
n(z)=\frac{N^{\prime}}{4 \pi D z} \exp \left\{-\frac{v}{D} z\right\}
$$

for a point directly in front of the release point and in the form

$$
n(z)=\frac{N^{\prime}}{4 \pi D z}
$$

for a point directly in the rear of the release point.

The diffusing fuel gas now causes attachment of free electrons at a rate

$$
\frac{d N_{e}}{d t}=-\beta n N_{e}+\delta\left(N_{+}-N_{e}\right)+D_{A} \nabla^{2} N_{e}
$$

where $\beta$ is the rate coefficient of attachment, $\delta$ is the rate coefficient of photodetachment, and $D_{A}$ is the coefficient for ambi-polar diffusion of electrons.

As a first approximation, electron diffusion may be neglected and the change of electron density expressed in the form

$$
N_{e}=N_{e o} \exp \left\{-\beta \int n d t\right\} .
$$

This equation is valid as long as the process of photodetachment can be neglected. If the time considered is long enough to allow an equilibrium between attachment and detachment to be established, the electron density is given by

$$
N_{e}=N_{e o} \frac{\delta}{\delta+\beta n} .
$$

Using (5) and the relation $z=-v \mathrm{t}$, we find

$$
\begin{aligned}
\int n d t & =-\int_{\infty}^{\xi} \frac{1}{v} n(z) d z \\
& =\frac{N^{\prime}}{4 \pi \mathrm{v} D} \int_{\xi}^{\infty} \frac{1}{z} \exp \left\{-\frac{v}{D} z\right\} d z \\
& =-\frac{N^{\prime}}{4 \pi v D} E_{i}\left(-\frac{v}{D} \xi\right)
\end{aligned}
$$

where $\xi$ is the distance from the release point to the point of measurement or the location of the antenna. $E_{i}(-x)$ is tabulated in tables [Jahnke and Emde, 1945].

The choice of numerical values for the parameters involved in the equations is guided by the fact that, after burnout, $7 \mathrm{lb}$ of fuel are left while the oxidizer

\begin{tabular}{|c|c|c|c|c|c|c|}
\hline$h$ & $n_{0}$ & $D$ & $v$ & $n(\xi)$ & $\int n d t$ & $N_{e} / N_{e o}$ \\
\hline 120 & 52.3 & 5.43 & 16.5 & 0.00 & 0.00 & 1.00 \\
\hline 140 & 7.52 & 54.0 & 15.5 & 3.42 & 0.55 & 0.76 \\
\hline 160 & 2.62 & 185 & 14.0 & 4.22 & 1.86 & .40 \\
\hline 180 & 1.35 & 382 & 12.5 & 2.75 & 2.12 & .35 \\
\hline 200 & 0.782 & 681 & 10.8 & 1.73 & 2.13 & .34 \\
\hline 220 & .480 & 1137 & 8.7 & 1.10 & 2.19 & .33 \\
\hline 240 & .307 & 1807 & 6.7 & 0.71 & 2.32 & .31 \\
\hline $\mathrm{km}$ & $\frac{10^{10}}{\mathrm{~cm}^{3}}$ & $10^{6} \frac{\mathrm{cm}^{2}}{\mathrm{sec}}$ & $10^{4} \frac{\mathrm{cm}}{\mathrm{sec}}$ & $\frac{10^{10}}{\mathrm{~cm}^{3}}$ & $10^{8} \frac{\mathrm{sec}}{\mathrm{cm}^{3}}$ & \\
\hline
\end{tabular}
has been used up. During ascent a release of the fuel at a rate of $17.3 \mathrm{~g} / \mathrm{sec}$ is reasonable. With a molecular weight of 93 this amounts to $1.12 \times 10^{23}$ particles per second. The diffusion coefficient is computed based on a model of rigid spheres with a collision distance $\sigma_{12}$ of $4.4 \times 10^{-8} \mathrm{~cm}$ [Chapman and Cowling, 1952]. Temperatures and densities are taken in accordance with the U.S. Standard Atmosphere 1962.1 A summary of the numerical results is shown in table 1 .

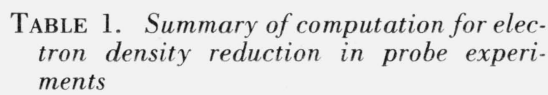
ments

Available from U.S. Government Printing Office, Washington, D.C. 20402. 
$\mathrm{h}$ is the height of the rocket

$n_{0}$ is the neutral particle density of the air

$D$ is the diffusion coefficient of the fuel in air

$v$ is the velocity of the rocket

$n(\xi)$ is the particle density of the fuel at a distance $\xi=680 \mathrm{~cm}$ in front of the release point.

$\int n d t$ is the time integrated fuel density, effective for attachment according to eq (10).

$N_{e} / N_{e o}$ is the electron density ratio computed with an attachment rate coefficient of $\beta=5 \times 10^{-9}$ $\frac{\mathrm{cm}^{3}}{\mathrm{sec}}$

The rate coefficient of attachment to the particular fuel is not known from laboratory measurements. The coefficient assumed here leads to an electron density decay which is in best agreement with the experimental results. The value of the coefficient is high and places the diffusing gas into the class of a very effective agent for attachment. Nevertheless, the value is not unreasonably high when one considers that a rate coefficient of $10^{-9}$ has been measured for attachment of sulfur hexafluoride $\left(\mathrm{SF}_{6}\right)$ [Hickam and Fox, 1956].

\section{Outgassing Effect on Propagation Experiments}

It can be assumed that outgassing occurred on all our previous Aerobee rocket flights. We used Aerobee rockets for a propagation experiment of the pulse delay type between the years 1953 and 1959 . Useful data were obtained from seven rockets launched from Holloman Missile Test Center, New Mexico, and three rockets launched from the Churchill range in

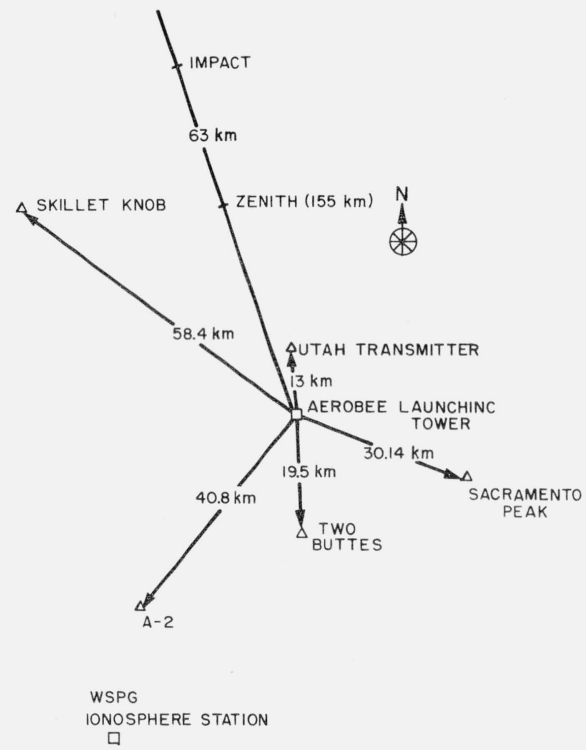

FIGURE 3. Geographical location of the ground transmitting and receiving sites for propagation experiments in New Mexico.
Canada. It can be expected that a propagation experiment will lead to an error in the determination of the ambient electron density if outgassing produces a hole in the ionosphere around and behind the rocket. In fact effects due to outgassing were observed under certain geometrical conditions of rocket and propagation paths. Figure 3 shows the geographical location of the ground transmitting and receiving sites with respect to the Aerobee launching tower and a typical flight trajectory for the facility in New Mexico. The experiment consisted of two parts [Pfister and Ulwick, 1958]. First, the time delay of the upward transmission on seven discrete stepped frequencies in the range from 4 to $5 \mathrm{Mc} / \mathrm{s}$ was telemetered back to the ground. Second, the time delay of the downward transmission on $6 \mathrm{Mc} / \mathrm{s}$ was measured at different receiving sites on the ground. In the earlier experiments the ground transmitter was located at Station Two Buttes and in order to achieve a more vertical propagation path later was transferred to the Utah site. Receivers were placed into several of the following sites: Army-2, Skillet Knob, Sac Peak, Two Buttes, and Utah.

Outgassing effects were observed only on rocket ascent and then only on propagation with the stations Two Buttes, Army-2, and Sac Peak. The effect consists of a reduction of the time delay of the pulse over a portion of the flight. It can be found when the undisturbed propagation path follows more or less closely the rocket trajectory. How closely depends on the frequency and the trapping capability of the hole. Interestingly, in an experiment using the downward transmission, no difference in the effect was observed at Two Buttes, where rocket and ray path practically coincide in the ionosphere, compared with Army-2 or Sac Peak where a bending of the ray path into the hole is necessary to be able to follow the rocket trajectory.

An example of the outgassing effect on pulse delay measurements is shown in figure 4. Aerobee Rocket No. 67 was launched in 1956 on June 13 at 1351 MST in New Mexico. An electron density profile was computed from the available undisturbed measurements: the downward transmission delay data at Skillet Knob for ascent and descent and the upward transmission delay data from Utah site for rocket descent. The mean electron density of the three sets of data was used to compute a theoretical undisturbed delay curve for the downward transmission at Army-2 on rocket ascent and descent.

This curve, together with the actual measurements, is displayed in figure 4. It clearly indicates a hole in the ionosphere along the propagation path during rocket ascent between 100 and $130 \mathrm{~km}$. Two additional traces of echo delays are seen. One is a reflection from a level just above the peak of the rocket trajectory. The interpretation of the other one is still open to speculation. Note that the apparent electron content is reduced to about 70 percent of the undisturbed value.

It is interesting to make a numerical estimate of the attachment effect. As in Aerobee SN-37 a fuel escape 


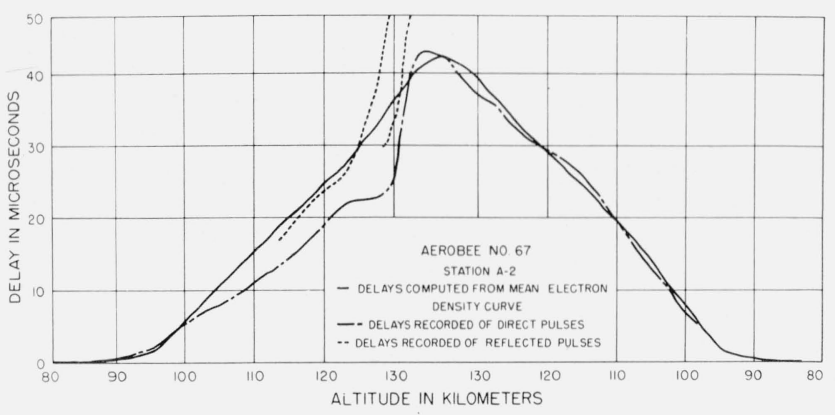

Figure 4. Comparison of experimental and theoretical delay data for Aerobee No. 67.

at a rate of $17.3 \mathrm{~g} / \mathrm{sec}$ may be assumed. The propagation experiment is affected by a reduction of electron densities over distances of the order of $10 \mathrm{~km}$. Therefore, the initial expansion of the fuel can be neglected and the density of the diffusing gas behind the rocket may be computed from (6). In addition the time is long enough that essentially an equilibrium between attachment and detachment processes is achieved. In this case the reduction of electron density is computed according to (9).

TABLE 2. Summary of computations for electron density reduction in propagation experiments

\begin{tabular}{c|c|c|c|c|r}
\hline \hline$h$ & $n_{0}$ & $D$ & $\xi$ & $n(\xi)$ & $\frac{\delta}{\beta n+\delta}$ \\
\hline 100 & 1040 & 0.21 & 46 & 0.925 & 0.19 \\
110 & 207 & 1.18 & 27.5 & .275 & .42 \\
120 & 52.3 & 5.43 & 15 & .109 & .65 \\
125 & 27.6 & 9.14 & 7.5 & .130 & .61 \\
\hline $\mathrm{km}$ & $\underline{10^{10}}$ & $10^{6} \frac{\mathrm{cm}^{2}}{\mathrm{sec}}$ & $10^{5} \mathrm{~cm}$ & $\underline{\mathrm{cm}^{10}}$ & \\
\hline
\end{tabular}

Rocket at $130 \mathrm{~km}$

$\xi=$ effective distance behind rocket

$\delta / \beta=0.2 \times 10^{10} \frac{\mathrm{l}}{\mathrm{cm}^{3}}$

Table 2 illustrates the attachment effect in the ionosphere behind the rocket for a rocket position at 130 $\mathrm{km}$ on ascent:

$\xi$ is the effective distance behind the rocket computed from the velocity of the rocket at the particular height and the flight time of the rocket between this height and $130 \mathrm{~km}$.

$n(\xi)$ is the particle density of the fuel when the rocket has reached $130 \mathrm{~km}$.

The reduction of electron density given by $\frac{\delta}{\delta+\beta n}$ was computed under the assumption of a value of $0.2 \times 10^{10}$ $\mathrm{cm}^{-3}$ for $\delta / \beta$. The choice of this value gives a reduction of the integrated electron density to about 50 percent. The time delay of the propagation experiment indicates a somewhat higher electron content. This is to be expected since the ray path, in following the hole in the ionosphere, is somewhat longer than

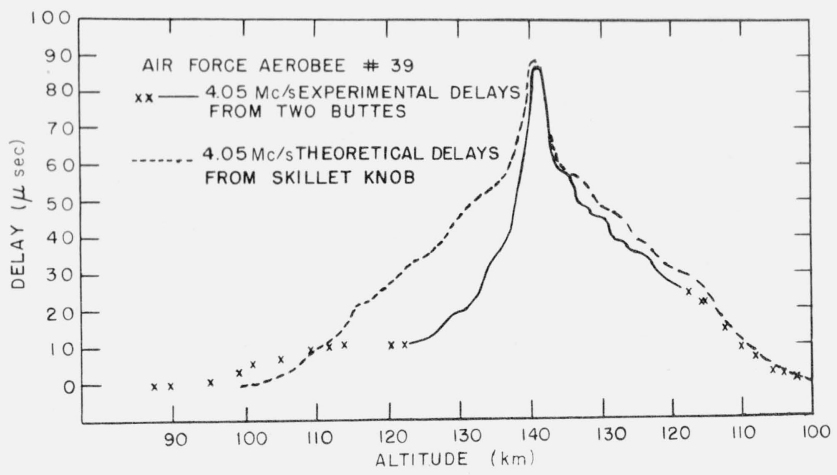

FigURE 5. Comparison of experimental and theoretical delay data for Aerobee No. 39.

for a horizontally stratified ionosphere. Introducing the attachment coefficient of $5 \times 10^{-9} \frac{\mathrm{cm}^{3}}{\mathrm{sec}}$, as deduced

previously from Aerobee Rocket SN-37, leads to a photodetachment rate of $10 / \mathrm{sec}$. Obviously these values are only estimates and not the result of a controlled experiment. The fact that the values are reasonable give confidence in the principal assumptions made for the outgassing process.

Another example of the effect is shown for Aerobee rocket No. 39 in figure 5. Part of the upward transmission data for this rocket has been analyzed previously [Lien et al., 1953 and 1954] in a rough preliminary fashion together with data from two $\mathrm{V}-2$ rockets and another Aerobee rocket. The conclusion at that time was a bifurcation of the $E$ layer. As seen from the figure, the slope of the experimental delay curve indicates a maximum of electron density at $98 \mathrm{~km}$ and a deep minimum at $115 \mathrm{~km}$. In the meantime a more complete analysis of the available data was carried out including the $6 \mathrm{Mc} / \mathrm{s}$ downward transmission data for both rocket ascent and descent recorded at Skillet Knob. Two electron density profiles were computed from the $6 \mathrm{Mc} / \mathrm{s}$ data which show reasonably good agreement between ascent and descent. A mean of the two profiles was used to compute a theoretical delay curve for the upward transmission from Two Buttes station, which is shown together with the measured delay curve in figure 5 . The crosses mark a spottiness in the measurements.

There again the delay data for rocket descent agree reasonably well, while for ascent a severe outgassing effect is indicated. The effect appears even more pronounced than for Aerobee No. 67. Peculiar is the increase of delay at low altitudes and the leveling off between 110 and $120 \mathrm{~km}$. There is no need to explain the increase in delay by an increase of electron density. A bending of the ray path into the hole might explain the increase in pulse delay and a subsequent widening of the hole and decrease in bending might explain the leveling off. The difference in frequency and possibly a slight difference in geometry probably is responsible for a different appearance of the outgassing effect shown on the two examples. 


\section{Observations With Solid-Fuel Rockets}

It is relatively simple to avoid effects of fuel outgassing as described before on Aerobee rockets or in fact on any rocket type using liquid fuel. The interruption of the flow of fuel with shutoff valves is the answer. Whenever this device was used no outgassing effects were observed.

The situation is different with solid-fuel rockets and the question remains to what extent outgassing might present a problem. As can be expected, each rocket motor type behaves differently. An example of experimental results is shown in figure 6 for an Astrobee 200 rocket, AA 15.196. This rocket is similar in performance to the Aerobee 150 rocket. It was launched from Eglin Air Force Base, Florida, in the summer of 1962 and carried a multiple ionospheric experiment. The instrumentation was described previously in detail [Ulwick et al., 1962]. Shown in figure is is an electron density profile derived from an ionosonde record. In substantial agreement with this curve is the positive ion density measured with a retarding potential probe in the front of the rocket. For the present purpose the low values below $120 \mathrm{~km}$ can be disregarded. They could be caused by a shielding effect of the ejected nose tip package. The electron density was measured with the standing wave imped-

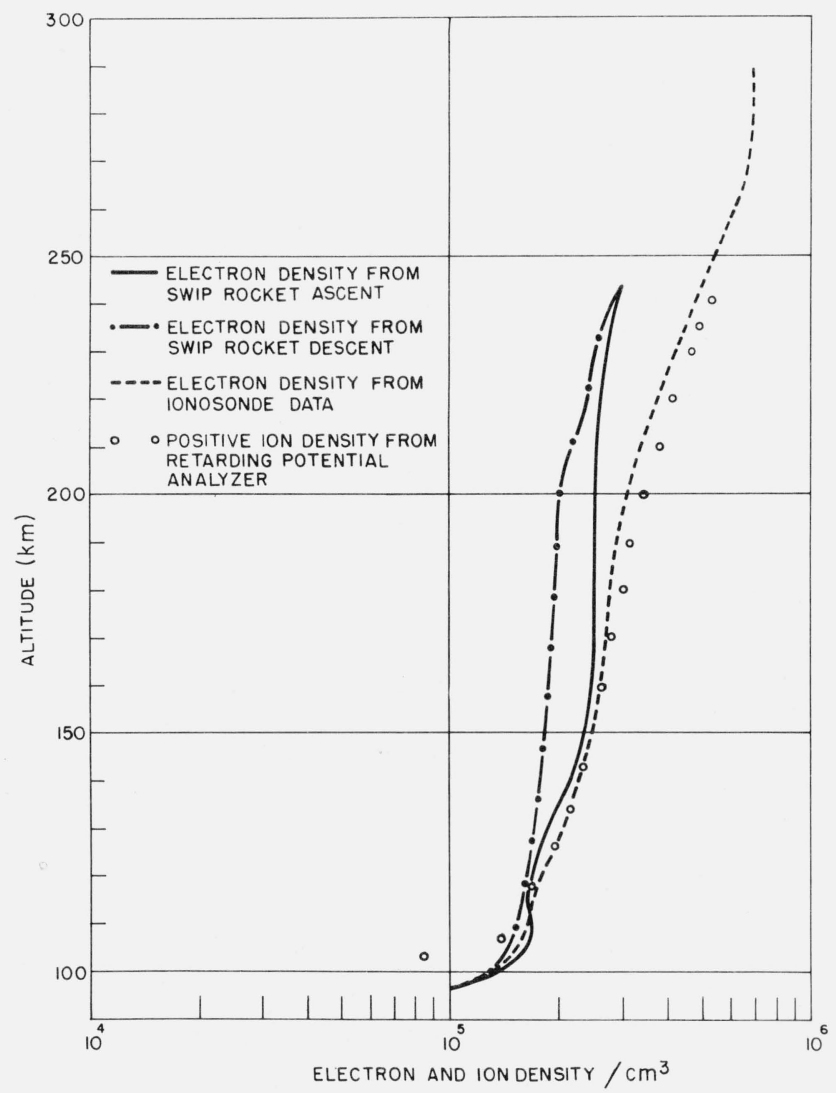

Figure 6. Results from Astrobee 200 AA 15.196, fired 30 April 1962, 1235 CST at Eglin Air Force Base, Florida. ance probe on ascent and descent and shows a typical outgassing effect. The reduction of electron density due to attachment is less severe than in the Aerobee rockets and the effect decreases towards the end of the rocket flight. Tipover of the rocket occurred below $100 \mathrm{~km}$.

In spite of the fact that the Astrobee 200 is an aerodynamically very well behaved rocket and that solidfuel rockets are ready to be fired any time into an unscheduled event, the outgassing effect prevented us from using this rocket type for environmental measurements in an auroral display as originally intended.

A brief summary of our experience with other types of solid-fuel rockets is as follows:

The Spaerobee rocket is an Aerobee rocket with an additional third stage driven by a solid-fuel motor. In a noontime firing in March 1961 we measured electron density between the $E$ layer and the peak of the $F_{2}$ layer with the standing wave impedance probe. No data are available from the retarding potential analyzer. The electron density agrees with ionosonde data within the instrumental error. The descent data are slightly above the level of the ascent data which is an evidence against outgassing from the motor. Somewhat suspicious is a rather larger spin modulation on the experimental curve which was also observed on satellite experiments and was interpreted there as a wake effect on the active half of the probe antenna [Haycock et al., 1964].

The Blue Scout D-5 is an all solid-fuel rocket with four stages. The last stage uses a ABL X-248 motor and reaches altitudes well above the peak of the $F_{2}$ layer. An experiment combining the impedance probe on 3 and $7 \mathrm{Mc} / \mathrm{s}$ for electron densities and a spherical ion trap for positive ions was flown in April 1961 around midnight up to an altitude of $1800 \mathrm{~km}$. In comparing the results for electrons and ions, good agreement was noticed [Sagalyn and Smiddy, 1964], and no effect was found which would indicate outgassing.

The Honest John-Nike-Nike combination can reach an altitude of about $150 \mathrm{~km}$. Several of these rockets were fired into a disturbed ionosphere at night carrying again an impedance probe and an ion trap experiment. One rocket in particular is suspected to be affected by outgassing. It shows a deficiency of electrons starting at $130 \mathrm{~km}$ on ascent and continuing through descent until rocket tipover at $90 \mathrm{~km}$. Due to the disturbed nature of the ionosphere and the preliminary nature of the analysis no definite conclusions can be drawn at the present time.

Nike-Cajun and Nike-Apache are rockets very often used for measurements in the $E$ layer. We ourselves are not able to report on experimental results. From our knowledge of results measured elsewhere no obvious outgassing effect has been noticed on these rockets.

The Black Brant is a heavy one-stage rocket which carries considerable payload up to $140 \mathrm{~km}$ or higher. A Black Brant rocket fired during the day in July 1963 from Churchill, Canada, carried several radio frequency probes and a retarding potential probe [Ulwick, 


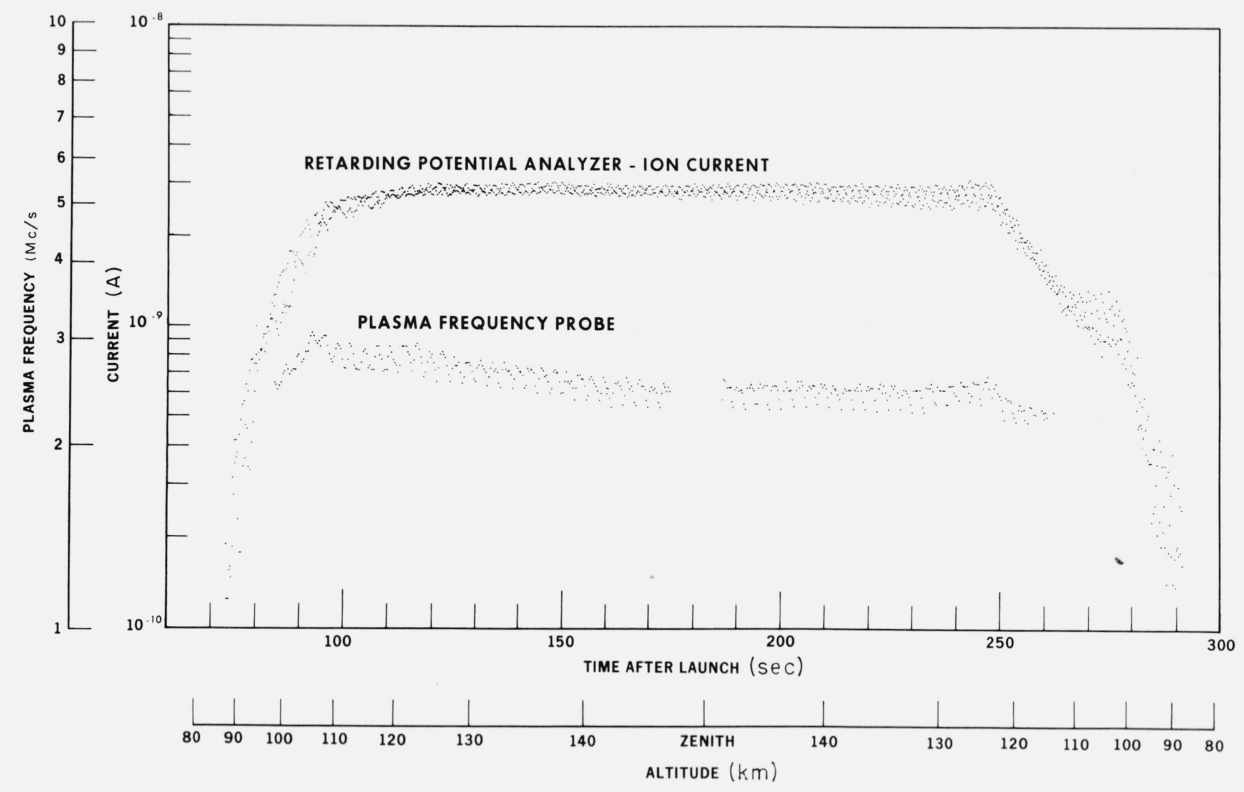

Figure 7. Results from Black Brant AB 17.601, fired 26 July 1963, 1615 CST at Churchill, Canada.

Pfister, and Haycock, 1963]. The detector for positive ions was mounted sideways and could measure only relative values of ion densities. Figure 7 shows a comparison between the positive ion current and the series resonance frequency of the plasma frequency probe. The series resonance frequency is slightly lower than the plasma frequency and the experimental data are of better quality. Both curves show a "beat" effect between the spin rate of the rocket and the sampling rate of the measurement. The ion current was sampled twice as fast as the resonance frequency. Unexplained in view of the extremely good stability of the rocket spin axis is the relatively large spin modulation of the electron density measurements. Rocket outgassing of the type discussed here is evident by the relative decrease of the measured electron density against the positive ion current in the course of the flight.

It is a pleasure to acknowledge the support of the following groups: The Aerospace Instrumentation Laboratory of Air Force Cambridge Research Laboratories for telemetry and vehicle integration support; and the research staffs of the University of Utah and Boston College Upper Air Research Laboratories for conducting the experiments and analyzing the data.

\section{References}

Chapman, S., and T. G. Cowling (1952), The Mathematical Theory of Non-uniform Gases (Cambridge Univ. Press, England).

Haycock, O. C., and K. D. Baker (1961), Measuring antenna impedance in the ionosphere, Electronics 34, 88-92.

Haycock, O. C., K. D. Baker, and J. C. Ulwick (1964), Experiences with the impedance probe on satellites, Proc. IEEE 52, 1029-1033.
Hickman, W. M., and R. E. Fox (1956), Electron attachment in sulfur hexafluoride using monoenergetic electrons, J. Chem. Phys. 25, No. 4, 643-647.

Hinteregger, H. (1960), Combined retarding potential analysis of photoelectron and environmental charged particles up to 234 km, Proc. First Intern. Space Science Symp., Nice, France (North Holland Publ. Co., Amsterdam, The Netherlands).

Jahnke, E., and F. Emde (1945), Tables of Functions (Dover Publishers, Inc., New York, N.Y.).

Lien, J. R., L. B. Linford, R. J. Marcon, J. C. Ulwick, D. R. McMorrow, and O. C. Haycock (1953), Bifurcation of the $E$ region, Phys. Rev. 92, 508.

Lien, J. R., R. J. Marcon, J. C. Ulwick, J. Aarons, and D. R. McMorrow (1954), Ionosphere research with rocket-borne instruments, Rocket Exploration of the Upper Atmosphere, ed. R. L. F. Boyd and M. Seaton, pp. 223-239 (Interscience Publishers, Inc., New York, N.Y.).

Pfister, W., and J. C. Ulwick (June 1958), The analysis of rocket experiments in terms of electron density distributions, J. Geophys. Res. 63, 315-333.

Pfister, W., J. C. Ulwick, and R. P. Vancour (1961), Some results of direct probing in the ionosphere, J. Geophys. Res. 66, No. 4, 1293-1297.

Pfister, W., and J. C. Ulwick (1961), Plasma sheath effects on rocket antenna, Proc. Am. Astronaut. Soc. Symp. (McGraw-Hill Book Co., Inc., New York, N.Y., 1965).

Sagalyn, R. C., and M. Smiddy (1964), Electrical processes in the nighttime exosphere, J. Geophys. Res. 69, No. 9, 1809-1823.

Ulwick, J. C., W. Pfister, R. P. Vancour, R. T. Bettinger, O. C. Haycock, and K. D. Baker (1962), Firing of an Astrobee 200 rocket with a multiple ionospheric experiment, Proc. IRE 50, No. 11, $2272-2286$.

Ulwick, J. C., W. Pfister, and O. C. Haycock (1963), RF probe measurements from a recent Churchill firing into an auroral absorption, Aeronomy Report No. 1, pp. 173-175 (University of Illinois).

Ulwick, J. C., W. Pfister, O. C. Haycock, and K. D. Baker (1964), Standing wave impedance probe, COSPAR Information Bull. No. 17, 117-146. 